\title{
On the Dynamics and Optimal Control of Constrained Mechanical Systems
}

\author{
Simeon Schneider, Peter Betsch
}

\author{
Institute of Mechanics \\ Karlsruhe Institute of Technology (KIT) \\ Otto-Ammann-Platz 9, 76131 Karlsruhe, Germany \\ simeon.schneider@kit.edu, peter.betsch@kit.edu
}

\begin{abstract}
The focus of this work is on optimal control in redundant coordinates with a special attention to the boundary constraints that arise in this context. Due to the similarity of the optimization problem of optimal control to the Lagrangian formalism of classical mechanics, this is considered first. Once the mechanical problem of the boundary conditions in redundant coordinates has been discussed, the knowledge gained is transferred to the optimal control problem in order to solve the problem in redundant coordinates. Finally, for each section the equivalence of the problem in minimal coordinates and redundant coordinates is shown by numerical results.
\end{abstract}

Keywords: Optimal control, boundary value problems, multibody dynamics

\section{INTRODUCTION}

Optimal control contains a large field of applications, from the optimal control of economical or chemical processes to the optimal control of robots or satellites. The latter ones can be summarized to mechanical systems and represent the focus of attention within this work.

There are several methods to describe the behaviour of the systems, the two most frequently chosen being the description of the complete system in minimal or redundant coordinates. However, depending on the choice of the description of the system, different types of mathematical systems of equations arise. As is well known, the description of the system in minimal coordinates yields a system of ordinary differential equations (ODEs), whereas the description of the system in redundant coordinates yields a system of differential algebraic equations (DAEs) for the equations of motion. Of course, both systems of equations describe the same motion of the mechanical system. Since the equations of motion are the constraints in the optimal control problem of the mechanical system, their description plays an essential role in the formulation of the optimal control problem. While the analytical and numerical solution of the optimal control problem in minimal coordinates can be considered as already well researched, this is not yet true for the description in redundant coordinates. However, since finding minimal coordinates can be difficult and in the worst case impossible, the focus of the present work lies on using DAEs as state equations in the optimal control problem. Due to the parallels between the Lagrangian formalism of classical mechanics and the optimization problem of optimal control, the Lagrangian formalism will be considered first. Subsequently, the obtained knowledge is applied to the optimal control problem, with special attention to the boundary constraints to be defined.

To keep it short and simple, the general approach will be illustrated with the example of a physical pendulum on a slide depicted in Figure 1.

\section{CONSTRAINED MECHANICS AND THE LAGRANGIAN FORMALISM}

In this section the boundary value problem (BVP) of the Lagrangian formalism will be considered with a special attention to the boundary conditions arising in redundant coordinates. Before starting with the description of the BVP in redundant coordinates, however, we will briefly discuss 
the BVP in minimal coordinates. Once the procedure is known, the BVP is derived in redundant coordinates and the problems which arise are discussed. Thereupon, a simple but promising way to solve the mechanical BVP in redundant coordinates is described. The equivalence of both boundary value problems under consideration is illustrated with a numerical example.

\subsection{The mechanical BVP in minimal coordinates}

Let $I \in\left[t_{0}, t_{f}\right]$ be a time interval, $q: I \rightarrow \mathscr{M}$ be the minimal coordinates on the configuration manifold $\mathscr{M}:=\left\{q \in \mathbb{R}^{2} \mid q_{1}=x_{1}, q_{2}=x_{6}\right\}$ of the system at hand (Fig. 1), and $\mathscr{T} \mathscr{M}$ be the tangent bundle, see for example [2]. Then the Lagrangian $L: \mathscr{T} \mathscr{M} \rightarrow \mathbb{R}$ is defined by

$$
L(q)=T(q, \dot{q})-V(q)=\frac{1}{2} \dot{q}^{a} g_{a b} \dot{q}^{b}-V(q)
$$

with $T: \mathscr{T} \mathscr{M} \rightarrow \mathbb{R}$ being the kinetic energy, $V: \mathscr{M} \rightarrow \mathbb{R}$ being the potential energy and $g_{a b}$ being the metric tensor, better known as mass matrix. Finally let $\mathbb{P} \in \mathscr{M} \times \mathscr{T}^{*} \mathscr{M}$ be the phase space of the mechanical system. Then using Hamilton's principle, the mechanical BVP is described by the optimization problem

Problem 1. Mechanical BVP in minimal coordinates (MBVPM)

Find the extremal curve $\gamma: I \rightarrow \mathbb{P}$ given by the action integral

$$
S^{M}(q)=\int_{t_{0}}^{t_{f}} L(q, \dot{q}) \mathrm{d} t
$$

Using Livens principle, see [3], [4], one may also set the equivalent problem to (1) by

Problem 2. Extended mechanical BVP in minimal coordinates (MBVPML)

Find the extremal curve $\gamma: I \rightarrow \mathbb{P}$ given by the action integral

$$
S^{M}(q, v, p)=\int_{t_{0}}^{t_{f}} L(q, v)-p_{a}\left(v^{a}-\dot{q}^{a}\right) \mathrm{d} t
$$

with $v \in \mathscr{T} \mathscr{M}$ and and $p \in \mathscr{T}^{*} \mathscr{M}$.

Taking the variation of (2) yields

$$
\delta S^{M}(q, v, p)=\int_{t_{0}}^{t_{f}}\left(\frac{\partial L(q, v)}{\partial v^{a}}-p_{a}\right) \delta v^{a}+\left(\frac{\partial L(q, v)}{\partial q^{a}}\right) \delta q^{a}+\left(\dot{q}^{a}-v^{a}\right) \delta p_{a}+p_{a} \delta \dot{q}^{a} \mathrm{~d} t
$$

Applying the integration by parts

$$
\int_{t_{0}}^{t_{f}} p_{a} \delta \dot{q}^{a} \mathrm{~d} t=\left[p_{a} \delta q^{a}\right]_{t_{0}}^{t_{f}}-\int_{t_{0}}^{t_{f}} \dot{p}_{a} \delta q^{a} \mathrm{~d} t
$$
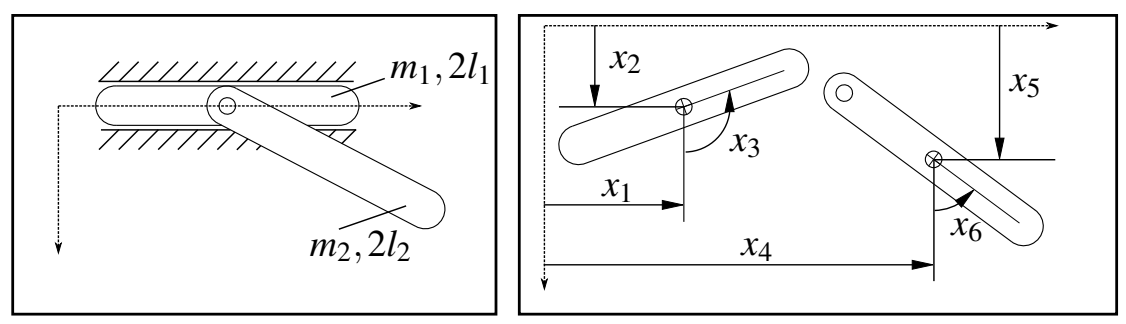

Figure 1. Multibody system of a physical pendulum on a moving slide taken from [1]. 
and inserting the Legendre transformation resulting from the variation $\delta v^{a}$, which yield the Legendre transformed function $H^{M}: \mathscr{T}^{*} M \rightarrow \mathbb{R}$ of the Lagrangian,

$$
H^{M}=\frac{1}{2} p_{a} g^{a b} p_{b}+V(q)
$$

finally yields

$$
\delta S^{M}(q, p)=\int_{t_{0}}^{t_{f}}\left(-\dot{p}_{a}-\frac{\partial H^{M}(q, p)}{\partial q^{a}}\right) \delta q^{a}+\left(\dot{q}^{a}-\frac{\partial H^{M}(q, p)}{\partial p_{a}}\right) \delta p_{a} \mathrm{~d} t+\left[p_{a} \delta q^{a}\right]_{t_{0}}^{t_{f}}
$$

Now, imposing the stationary condition $\delta S^{M}(q, p)=0$, one obtains the necessary optimality conditions

$$
\begin{aligned}
\dot{q}^{a} & =\frac{\partial H^{M}(q, p)}{\partial p_{a}} \\
\dot{p}_{a} & =-\frac{\partial H^{M}(q, p)}{\partial q^{a}}
\end{aligned}
$$

along with the boundary conditions

$$
0=\left[p_{a} \delta q^{a}\right]_{t_{0}}^{t_{f}}
$$

Note that, at this stage, we do not impose the common end-point conditions on $\delta q^{a}$, but rather keep the boundary conditions leading to a BVP comprised of (7) and (8).

\subsection{The mechanical BVP in redundant coordinates}

Let $x: I \rightarrow \mathscr{M}$ be the description of the mechanical system in redundant coordinates and thus $\mathscr{M}:=\left\{x \in \mathbb{R}^{6} \mid g^{r}(x)=0\right\}$. Then the Lagrangian in redundant coordinates may be defined by

$$
\hat{L}(x, y)=T(x, \dot{x})-V(x)-y_{r} g^{r}(x)=\frac{1}{2} \dot{x}^{i} \hat{g}_{i a} \dot{x}^{j}-V(x)-y_{r} g^{r}(x)
$$

In analogy to the last section we obtain

\section{Problem 3. Extended mechanical BVP in redundant coordinates (MBVPRL)}

Find the extremal curve $\gamma: I \rightarrow \mathbb{P}$ given by the action integral

$$
S^{M}(x, \hat{v}, \hat{p}, y)=\int_{t_{0}}^{t_{f}} \hat{L}(x, \hat{v}, y)-\hat{p}_{i}\left(\hat{v}^{i}-\dot{x}^{i}\right) \mathrm{d} t
$$

Following the steps which led to (3) - (6), we obtain

$$
\delta S^{M}(x, \hat{p}, y)=\int_{t_{0}}^{t_{f}}\left(\frac{\partial \hat{H}(x, \hat{p}, y)}{\partial x^{i}}-\dot{\hat{p}}_{i}\right) \delta x^{i}+\left(\dot{x}^{i}-\frac{\partial \hat{H}(x, \hat{p}, y)}{\partial \hat{p}_{i}}\right) \delta \hat{p}_{i}+g^{r}(x) \delta y_{r} \mathrm{~d} t+\left[\hat{p}_{i} \delta x^{i}\right]_{t_{0}}^{t_{f}}
$$

where the Hamiltonian $\hat{H}^{M}$ in redundant coordinates is given by

$$
\hat{H}^{M}(x, \hat{p}, y)=\hat{p}_{i} \hat{g}^{i j} \hat{p}_{j}+V(x)+y_{r} g^{r}(x)
$$

Thus, the mechanical BVP in redundant coordinates is described by the necessary optimality conditions

$$
\begin{aligned}
\dot{x}^{i} & =\frac{\partial \hat{H}^{M}(x, \hat{p}, y)}{\partial p_{i}} \\
\dot{\hat{p}}_{i} & =-\frac{\partial \hat{H}^{M}(x, \hat{p}, y)}{\partial x^{i}} \\
0 & =\frac{\partial \hat{H}^{M}(x, \hat{p}, y)}{\partial y_{r}}
\end{aligned}
$$


along with the boundary conditions

$$
0=\left[\hat{p}_{i} \delta x^{i}\right]_{t_{0}}^{t_{f}}
$$

However, due to the constraints $g^{r}(x)=0$ associated with (13) 3 , the variations $\delta x$ are not independent and shall be further investigated with respect to the boundary conditions. Since (13) gives rise to index-3 DAEs, see [5], it is well known, that the implicit definition of $y_{r}$ is given by

$$
\frac{\mathrm{d}^{2}}{\mathrm{~d} t^{2}}\left(g^{r}(x)\right)=G^{r}(x, p, y)
$$

However, since either $x^{i}$ or $p_{i}$ is properly defined on the boundary, $y_{r}$ is in general unknown on the boundaries, until the solution is known. Thus $g^{r}(x(t))=0$ holds on the boundaries, which reduces the independent redundant coordinates to $a$ and thus only $a$ boundary conditions may be defined with respect to the variation of $x$ on the boundaries. To answer the question of properly set admissible boundaries, it is helpful to decompose the redundant coordinates into their normal and tangential parts. This is to happen in the following section.

\subsubsection{Decomposed vector spaces}

Let $\mathscr{B}_{\hat{V}_{p}}$ and $\mathscr{B}_{\hat{V}_{p}^{*}}$ be the bases of the local vector spaces $\hat{V}_{p}$ and $\hat{V}_{p}^{*}$ at point $p$ on $\mathscr{M}$ in redundant coordinates. Let further $\mathscr{B}_{\mathscr{T}_{p} \mathscr{M}}$ and $\mathscr{B}_{\mathscr{T}_{p}^{*} \mathscr{M}}$ be the bases of the tangential spaces $\mathscr{T}_{p} \mathscr{M}$ and $\mathscr{T}_{p}^{*} \mathscr{M}$, and $\mathscr{B}_{\mathscr{N}_{p} \mathscr{M}}$ and $\mathscr{B}_{\mathscr{N}_{p}^{*} \mathscr{M}}$ be the bases of the normal spaces $\mathscr{N}_{p} \mathscr{M}$ and $\mathscr{N}_{p}^{*} \mathscr{M}$ at $p$. Now let's assume, the mapping $n^{r}(x)=$ const. exists and thus $g^{r}\left(n^{s}(x)\right)=0$ holds. Since the constraints $g^{r}(x)$ are known in redundant coordinates, following [1], see also [6], [7] and [8], a straight forward calculation yields the metric tensors associated with the normal and tangential spaces, $\left(\hat{g}_{r s}, \hat{g}^{r s}\right)$, and $\left(g_{a b}, g^{a b}\right)$, respectively, along with the Jacobians

$$
\begin{aligned}
& \frac{\partial x^{i}}{\partial n^{r}}: \hat{V}_{p} \rightarrow \mathscr{N}_{p} \mathscr{M}, \quad \frac{\partial n^{r}}{\partial x^{i}}: \hat{V}_{p}^{*} \rightarrow \mathscr{N} *_{p} \mathscr{M} \\
& \frac{\partial x^{i}}{\partial q^{a}}: \hat{V}_{p} \rightarrow \mathscr{T}_{p} \mathscr{M}, \quad \frac{\partial q^{a}}{\partial x^{i}}: \hat{V}_{p}^{*} \rightarrow \mathscr{T} *_{p} \mathscr{M}
\end{aligned}
$$

Now let the bases of the decomposed local vector space $V_{p}:=\mathscr{N}_{p} \mathscr{M} \cup \mathscr{T}_{p} \mathscr{M}$ and its dual space $V_{p}^{*}:=\mathscr{N} *_{p} \mathscr{M} \cup \mathscr{T} *_{p} \mathscr{M}$ be defined by $\mathscr{B}_{V_{p}}$ and $\mathscr{B}_{V_{p}^{*}}$, whereat by definition

$$
\mathscr{B}_{\mathscr{T}_{p} \mathscr{M}} \perp \mathscr{B}_{\mathscr{N}_{p} \mathscr{M}}, \quad \mathscr{B}_{\mathscr{T}_{p}^{*} \mathscr{M}} \perp \mathscr{B}_{\mathscr{N}_{p}^{*} \mathscr{M}}
$$

holds. This leads to the mappings

$$
\begin{aligned}
& \frac{\partial x^{j}}{\partial z^{i}}: \hat{V}_{p} \rightarrow \mathscr{N}_{p} \mathscr{M} \cup \mathscr{T}_{p} \mathscr{M} \\
& \frac{\partial z^{j}}{\partial x^{i}}: \hat{V}_{p}^{*} \rightarrow \mathscr{N} *_{p} \mathscr{M} \cup \mathscr{T} *_{p} \mathscr{M}
\end{aligned}
$$

where the coordinates $z^{i}=\left\{n^{r}, q^{a}\right\}$ and momenta $p_{i}=\left\{p_{r}, p_{a}\right\}$ have been introduced.

\section{Remark. Velocity components in normal directions}

Since

$$
\frac{\mathrm{d} g^{r}(x)}{\mathrm{d} t}=\frac{\partial g^{r}(x)}{\partial n^{s}} \dot{n}^{s}=0
$$

and $\frac{\partial g^{r}(x)}{\partial n^{s}} \neq 0$ in general, it can be seen that $\dot{n}^{s}=0$ holds. 


\subsubsection{The boundary conditions in redundant coordinates}

Using the relationships

$$
\begin{gathered}
\delta x^{i}=\frac{\partial x^{i}}{\partial z^{j}} \delta z^{j}=\frac{\partial x^{i}}{\partial n^{r}} \delta n^{r}+\frac{\partial x^{i}}{\partial q^{a}} \delta q^{a} \\
\hat{p}_{i}=\frac{\partial z^{j}}{\partial x^{i}} p_{j}=\frac{\partial n^{r}}{\partial x^{i}} p_{r}+\frac{\partial q^{a}}{\partial x^{i}} p_{a}
\end{gathered}
$$

inserting them into (11) and making use of $\dot{n}^{r}=p_{r}=0$ leads to the necessary optimality conditions in decomposed coordinates given by

$$
\begin{aligned}
\dot{q}^{a} & =\frac{\partial H^{M}(q, n, p)}{\partial p_{a}} \\
0 & =-\frac{1}{2} p_{a} \frac{\partial g^{a b}}{\partial n^{r}} p_{b}-\frac{\partial V}{\partial n^{r}}-y_{r} \\
\dot{p}_{a} & =-\frac{\partial H^{M}(q, n, p)}{\partial q^{a}} \\
0 & =g^{r}(x)
\end{aligned}
$$

with the Hamiltonian being defined by (5). For the boundary conditions follow

$$
\begin{aligned}
0 & =\left[p_{i} \frac{\partial x^{i}}{\partial n^{r}} \delta n^{r}\right]_{t_{0}}^{t_{f}}+\left[p_{i} \frac{\partial x^{i}}{\partial q^{a}} \delta q^{a}\right]_{t_{0}}^{t_{f}} \\
& =\left[p_{r} \delta n^{r}\right]_{t_{0}}^{t_{f}}+\left[p_{a} \delta q^{a}\right]_{t_{0}}^{t_{f}} \\
& =\left[p_{a} \delta q^{a}\right]_{t_{0}}^{t_{f}}
\end{aligned}
$$

since $p_{r}=0$ everywhere.

Remark. The implicit definitions of $y_{r}$

It can be seen from (24b) that $r$ ODEs in redundant coordinates correspond to the implicit definition of $y_{r}$.

Remark. The duality of the derivative of the constraints and the variation for $\delta n^{r}$

A straight forward calculation yields

$$
\frac{\mathrm{d}^{2}}{\mathrm{~d} t^{2}}\left(g^{r}(x)\right)=G^{r}(x, \hat{p}, y)=g^{r s} G_{s}(q, n, p, y)
$$

with

$$
G_{r}(q, n, p, y)=-\frac{1}{2} p_{a} \frac{\partial g^{a b}}{\partial n^{r}} p_{b}-\frac{\partial V}{\partial n^{r}}-y_{r}
$$

Writing (25) in terms of redundant coordinates finally yields the proper BVP in redundant coordinates, defined by the necessary optimality conditions (13) and the boundary conditions

$$
0=\left[\hat{p}_{i} \frac{\partial x^{i}}{\partial q^{a}} \delta q^{a}\right]_{t_{0}}^{t_{f}}
$$

Remark. Setting boundary conditions by using Lagrangian multipliers

The natural boundary conditions (28) may also be augmented with suitable end-point conditions by using Lagrangian multipliers in the action integral. For example, this leads to

$$
S^{M}(\cdot)=\int_{t_{0}}^{t_{f}} L(q, \dot{q}) \mathrm{d} t+\mu_{a}^{0}\left(q^{a}\left(t_{0}\right)-\bar{q}_{0}^{a}\right)+\mu_{a}^{N}\left(q^{a}\left(t_{f}\right)-\bar{q}_{N}^{a}\right)
$$

and

$$
S^{M}(\cdot)=\int_{t_{0}}^{t_{f}} L(x, \dot{x}, y) \mathrm{d} t+\mu_{a}^{0} \frac{\partial q^{a}}{\partial x^{i}}\left(x^{i}\left(t_{0}\right)-\bar{x}_{0}^{i}\right)+\mu_{a}^{N} \frac{\partial q^{a}}{\partial x^{i}}\left(x^{i}\left(t_{f}\right)-\bar{x}_{N}^{i}\right)
$$

where $\bar{q}_{0}^{a}, \bar{q}_{N}^{a}$ and $\bar{x}_{0}^{i}, \bar{x}_{N}^{i}$, respectively, are prescribed coordinates. 


\subsection{Numerical example of the mechanical BVP}

In the following, the BVP of the mechanical system depicted in Fig. 1 is solved in minimal coordinates and redundant coordinates to show the equivalence of the BVPs numerically. As can be observed from Fig. 1, the constraints are specified by

$$
\begin{aligned}
& g^{1}(x)=x^{2} \\
& g^{2}(x)=x^{3} \\
& g^{3}(x)=x^{1}-x^{4}+l_{2} \sin \left(x^{6}\right) \\
& g^{4}(x)=x^{2}-x^{5}+l_{2} \cos \left(x^{6}\right)
\end{aligned}
$$

Let the potential energy in redundant and minimal coordinates be defined by

$$
V(x)=-m_{1} g x^{2}-m_{2} g x^{5}, \quad V(q)=-m_{2} g l_{2} \cos \left(q^{2}\right)
$$

with $g$ being the gravitational constant. Let further the kinetic energy be given by

$$
T(x, p)=\frac{1}{2} p^{i} \hat{g}_{i j} p^{j}, \quad T(q, n, p)=\frac{1}{2} p^{a} g_{a b} p^{b}
$$

with the metric tensors

$$
\hat{g}_{i j}=\hat{\mathbf{M}}=\left[\begin{array}{cccccc}
m_{1} & 0 & 0 & 0 & 0 & 0 \\
0 & m_{1} & 0 & 0 & 0 & 0 \\
0 & 0 & \frac{m_{1}\left(2 l_{1}\right)^{2}}{12} & 0 & 0 & 0 \\
0 & 0 & 0 & m_{2} & 0 & 0 \\
0 & 0 & 0 & 0 & m_{2} & 0 \\
0 & 0 & 0 & 0 & 0 & \frac{m_{2}\left(2 l_{2}\right)^{2}}{12}
\end{array}\right]
$$

and

$$
g_{a b}=\mathbf{M}=\left[\begin{array}{cc}
m_{1}+m_{2} & m_{2} l_{2} \cos \left(q^{2}\right) \\
m_{2} l_{2} \cos \left(q^{2}\right) & \frac{4}{3} m_{2} l_{2}^{2}
\end{array}\right]
$$

Applying the midpoint rule to the differential part, with step size $h=t_{n+1}-t_{n}$, finally yields the discrete necessary optimality conditions in redundant coordinates as

$$
\begin{aligned}
x_{n+1}^{i}-x_{n}^{i} & =h \frac{\partial H^{M}}{\partial \hat{p}_{i}^{n+\frac{1}{2}}} ; & n & =\{0,1, \ldots, N-1\} \\
\hat{p}_{i}^{n+1}-\hat{p}_{i}^{n} & =-h \frac{\partial H^{M}}{\partial x^{i}} ; & n & =\{0,1, \ldots, N-1\} \\
0 & =g^{r}\left(x_{n}\right) ; & n & =\{0,1, \ldots, N\}
\end{aligned}
$$

along with the boundary conditions

$$
\begin{array}{rlrl}
\frac{\partial q_{0}^{a}}{\partial x_{0}^{i}}\left(x_{0}^{i}-\bar{x}_{0}^{i}\right) \delta \mu_{a}^{0} & =0 ; & & \frac{\partial q_{N}^{a}}{\partial x_{N}^{i}}\left(x_{N}^{i}-\bar{x}_{N}^{i}\right) \delta \mu_{a}^{N}=0 \\
\left(\frac{\partial x_{0}^{i}}{\partial q_{0}^{a}} \hat{p}_{i}^{0}-\mu_{a}^{0}\right) \delta q_{0}^{a}=0 ; & \left(\frac{\partial x_{N}^{i}}{\partial q_{N}^{a}} \hat{p}_{i}^{N}-\mu_{a}^{N}\right) \delta q_{N}^{a}=0
\end{array}
$$

Since the implicit definition of $y_{r}$ can't be done properly on the boundary by the discrete system of ODEs, the implicit definitions are enforced by making use of (15). Thus, the additional boundary conditions

$$
G^{r}\left(x_{0}, \hat{p}_{0}, y_{0}\right)=0 ; \quad G^{r}\left(x_{N}, \hat{p}_{N}, y_{N}\right)=0
$$




$$
\begin{aligned}
\bar{x}\left(t_{0}\right) & =\left[0,0,0,1,0, \frac{\pi}{2}\right]^{T} \\
\bar{x}\left(t_{f}\right) & =\left[0,0,0,-1,0,-\frac{\pi}{2}\right]^{T} \\
\left(m_{1}, m_{2}\right) & =(1,1) \\
\left(l_{1}, l_{2}\right) & =(1,1) \\
T & =1.8 \\
N & =100
\end{aligned}
$$

Table 1. Specified boundary conditions as well as physical and geometric parameters of the mechanical system.
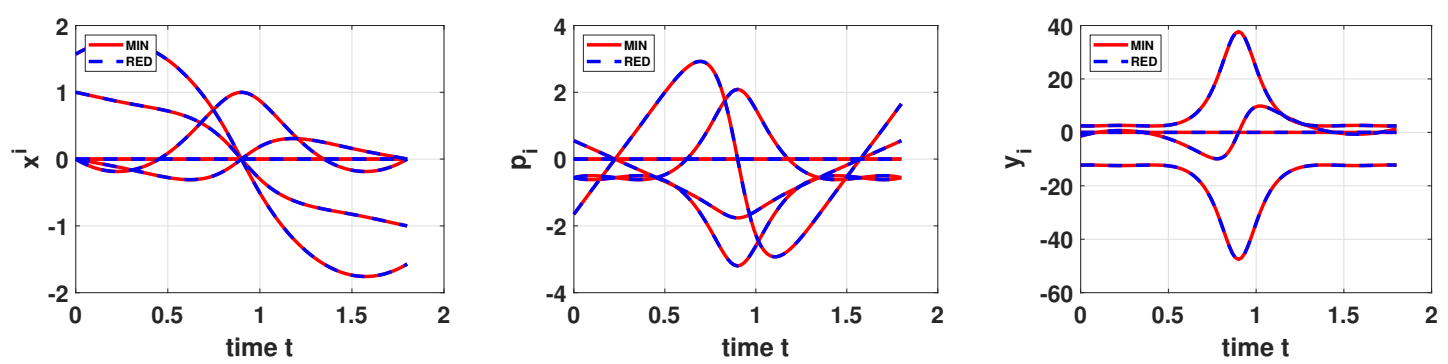

Figure 2. Solutions of the mechanical boundary value problem defined by Table 1 . Here the positions (left) and momenta (center) are shown in redundant coordinates. The dual quantities $y_{r}$ in minimal coordinates are calculated by making use of (24b)

are enforced which ensure the definition of $y_{r}$ on the boundaries.

For the discrete necessary optimality conditions in minimal coordinates follows

$$
\begin{array}{lll}
q_{n+1}^{a}-q_{n}^{a}=h \frac{\partial H^{M}}{\partial p_{a}^{n+\frac{1}{2}}} ; & n=\{0,1, \ldots, N-1\} \\
p_{a}^{n+1}-p_{a}^{n}=-h \frac{\partial H^{M}}{\partial q_{n+\frac{1}{2}}^{a}} ; & n=\{0,1, \ldots, N-1\}
\end{array}
$$

along with the boundary conditions

$$
\begin{aligned}
& \left(q_{0}^{a}-\bar{q}_{0}^{a}\right) \delta \mu_{a}^{0}=0 ; \quad\left(q_{N}^{a}-\bar{q}_{N}^{a}\right) \delta \mu_{a}^{N}=0 \\
& \left(p_{a}^{0}-\mu_{a}^{0}\right) \delta q_{0}^{a}=0 ; \quad\left(p_{a}^{N}-\mu_{a}^{N}\right) \delta q_{N}^{a}=0
\end{aligned}
$$

The boundary conditions chosen for the example, the length and mass of the slide and the pendulum as well as the length of the time interval $I \in[0, T]$ and the number of discrete time intervals, $N$, is shown in Table 1

Comparing the solutions depicted in Fig. 2, it can be seen that the BVPs are indeed equivalent.

Fig. 3 also shows a sequence of the solution of the mechanical boundary value problem.

\section{OPTIMAL CONTROL OF CONSTRAINED MECHANICAL SYSTEMS USING RE- DUNDANT COORDINATES}

In this section the BVP arising from the optimal control problem shall be treated by using redundant coordinates. Therefore, the well known optimal control problem in minimal coordinates will be discussed briefly to show the close connection between the Lagrange formalism in the mechanical BVP and the optimal control problem. Afterwards the necessary optimality conditions for the optimal control problem in redundant coordinates proofed in [9] shall be viewed. The boundary 

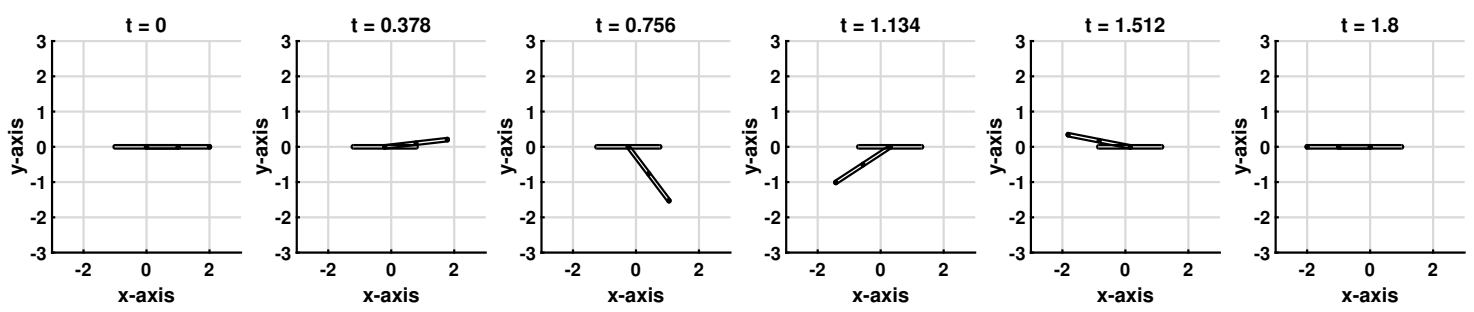

Figure 3. Snapshots of the motion resulting from the BVP with data given in Table 1.

conditions for the redundant coordinates will be described and finally the equivalence between the BVPs in terms of redundant and minimal coordinates will be shown with a numerical example.

\subsection{The optimal control BVP in minimal coordinates}

Let the controlled equations of motion be defined by (7) together with the control forces $u: I \rightarrow \mathbb{R}^{2}$ and thus

$$
\dot{q}^{i}=\frac{\partial H^{M}}{\partial p_{a}}, \quad \dot{p}_{a}=-\frac{\partial H^{M}}{\partial q^{a}}+u_{a}
$$

Then the standard optimal control problem reads

Problem 4. Optimal control problem in minimal coordinates (OCBVPM)

Minimize

$$
\mathbb{S}^{O C}(q, p, u)=\int_{t_{0}}^{t_{f}} C(q, p, u) \mathrm{d} t
$$

subject to

$$
\begin{aligned}
\dot{q}^{a} & =\frac{\partial H^{M}}{\partial p_{a}} \\
\dot{p}_{a} & =-\frac{\partial H^{M}}{\partial q^{a}}+u_{a}
\end{aligned}
$$

Augmenting the objective function of the optimization problem with the dynamic constraints (43) yields

\section{Problem 5. Augmented optimization problem in minimal coordinates (OCBVPML)}

Extremize

$$
\begin{aligned}
\mathbb{S}^{O C}\left(q, p, u, \lambda^{q}, \lambda_{p}\right) & =\int_{t_{0}}^{t_{f}} C(q, p, u)+\lambda_{a}^{q}\left(\dot{q}^{a}-\frac{\partial H^{M}}{\partial p_{a}}\right)+\lambda_{p}^{a}\left(\dot{p}_{a}-\left(-\frac{\partial H^{M}}{\partial q^{a}}+u_{a}\right)\right) \mathrm{d} t \\
& =\int_{t_{0}}^{t_{f}} \lambda_{a}^{q} \dot{q}^{a}+\lambda_{p}^{a} \dot{p}_{a}-\mathbb{H}^{O C}(\cdot) \mathrm{d} t
\end{aligned}
$$

Here, the Hamiltonian of the optimal control problem

$$
\mathbb{H}^{O C}\left(q, p, u, \lambda^{q}, \lambda_{p}\right)=\lambda_{a}^{q} \frac{\partial H^{M}}{\partial p_{a}}+\lambda_{p}^{a}\left(-\frac{\partial H^{M}}{\partial q^{a}}+u_{a}\right)-C(q, p, u)
$$


hast been introduced, see e.g. [8], [10] , [11]. Following the Lagrangian formalism by taking the variation and then applying integration by parts for the terms $\lambda_{i}^{q} \delta \dot{x}^{i}$ and $\lambda_{p}^{a} \delta \dot{p}_{a}$ finally yields

$$
\begin{aligned}
\delta \mathbb{S}^{O C}\left(q, p, u, \lambda^{q}, \lambda_{p}\right)= & \int_{t_{0}}^{t_{f}} \delta \lambda_{a}^{q}\left(\dot{q}^{a}-\frac{\partial \mathbb{H}^{O C}(\cdot)}{\partial \lambda_{a}^{q}}\right)+\delta \lambda_{p}^{a}\left(\dot{p}_{a}-\frac{\partial \mathbb{H}^{O C}(\cdot)}{\partial \lambda_{p}^{a}}\right) \\
& +\delta q^{a}\left(-\dot{\lambda}_{a}^{q}-\frac{\partial \mathbb{H}^{O C}(\cdot)}{\partial q^{a}}\right)+\delta p_{a}\left(-\dot{\lambda}_{p}^{a}-\frac{\partial \mathbb{H}^{O C}(\cdot)}{\partial p_{a}}\right)+\delta u_{a} \frac{\partial \mathbb{H}^{O C}(\cdot)}{\partial u_{a}} \mathrm{~d} t \\
& +\left[\lambda_{a}^{q} \delta q^{a}\right]_{t_{0}}^{t_{f}}+\left[\lambda_{p}^{a} \delta p_{a}\right]_{t_{0}}^{t_{f}}
\end{aligned}
$$

Thus, the necessary optimality conditions using minimal coordinates yield

$$
\begin{array}{rlrl}
\dot{q}^{a} & =\frac{\partial \mathbb{H}^{O C}(\cdot)}{\partial \lambda_{a}^{q}}, & \dot{\lambda}_{a}^{q}=-\frac{\partial \mathbb{H}^{O C}(\cdot)}{\partial q^{a}} \\
\dot{p}_{a}=\frac{\partial \mathbb{H}^{O C}(\cdot)}{\partial \lambda_{p}^{a}}, & \dot{\lambda}_{p}^{a}=-\frac{\partial \mathbb{H}^{O C}(\cdot)}{\partial p_{a}} \\
0=\frac{\partial \mathbb{H}^{O C}(\cdot)}{\partial u_{a}} &
\end{array}
$$

along with the boundary conditions

$$
0=\left[\lambda_{a}^{q} \delta q^{a}\right]_{t_{0}}^{t_{f}}, \quad 0=\left[\lambda_{p}^{a} \delta p_{a}\right]_{t_{0}}^{t_{f}}
$$

\subsection{The necessary optimality conditions in redundant coordinates}

Using redundant coordinates for the controlled equations of motion, the optimal control problem under investigation is given by

Problem 6. Optimal control problem in redundant coordinates (OCBVPR)

Minimize

$$
\hat{\mathbb{S}}^{O C}(x, \hat{p}, y, \hat{u})=\int_{t_{0}}^{t_{f}} C(x, \hat{p}, y, \hat{u}) \mathrm{d} t
$$

subject to

$$
\begin{aligned}
\dot{x}^{i} & =\frac{\partial \hat{H}^{M}}{\partial \hat{p}_{i}} \\
\dot{\hat{p}}_{i} & =-\frac{\partial \hat{H}^{M}}{\partial x^{i}}+\hat{u}_{i} \\
0 & =g^{r}(x)
\end{aligned}
$$

with $\hat{u}: I \rightarrow \mathbb{R}^{6}$. Again augmenting the objective function with the dynamic constraints (51) yields

$$
\begin{aligned}
\overline{\mathbb{S}}^{O C}\left(x, \hat{p}, y, \hat{u}, \hat{\lambda}^{q}, \hat{\lambda}_{p}, \eta\right) & =\int_{t_{0}}^{t_{f}} C(x, \hat{p}, y, u)+\hat{\lambda}_{i}^{q}\left(\dot{x}^{i}-\frac{\partial \hat{H}^{M}}{\partial \hat{p}_{i}}\right)+\hat{\lambda}_{p}^{i}\left(\dot{\hat{p}}_{i}-\left(-\frac{\partial \hat{H}^{M}}{\partial x^{i}}+\hat{u}_{i}\right)\right)+\eta_{r} g^{r}(x) \mathrm{d} t \\
& =\int_{t_{0}}^{t_{f}} \hat{\lambda}_{i}^{q} \dot{q}^{i}+\hat{\lambda}_{q}^{i} \dot{\hat{p}}_{i}-\overline{\mathbb{H}}^{O C}\left(x, \hat{p}, y, \hat{u}, \hat{\lambda}^{q}, \hat{\lambda}_{p}, \eta\right) \mathrm{d} t
\end{aligned}
$$

with

$$
\overline{\mathbb{H}}^{O C}\left(x, \hat{p}, y, \hat{u}, \hat{\lambda}^{q}, \hat{\lambda}_{p}, \eta\right)=\hat{\lambda}_{i}^{q} \frac{\partial \hat{H}^{M}}{\partial \hat{p}_{i}}+\hat{\lambda}_{p}^{i}\left(-\frac{\partial \hat{H}^{M}}{\partial x^{i}}+\hat{u}_{i}\right)-\eta_{r} g^{r}(x)-C(x, \hat{p}, y, u)
$$


One might now again demand

$$
\delta \overline{\mathbb{S}}^{O C}\left(x, \hat{p}, y, \hat{u}, \hat{\lambda}^{q}, \hat{\lambda}_{p}, \eta\right)=0
$$

to get the necessary optimality conditions. However, it is proofed in [9] (see also [10]), that this approach is not feasible. Instead, following [9], the proper necessary optimality conditions for the optimal control problem in redundant coordinates are given by

$$
\begin{array}{rlrl}
\dot{x}^{i} & =\frac{\partial \hat{\mathbb{H}}^{O C}(\cdot)}{\partial \hat{\lambda}_{i}^{q}}, & \dot{\hat{\lambda}}_{i}^{q}=-\frac{\partial \hat{\mathbb{H}}^{O C}(\cdot)}{\partial x^{i}} \\
\dot{\hat{p}}_{i}=\frac{\partial \hat{\mathbb{H}}^{O C}(\cdot)}{\partial \hat{\lambda}_{p}^{i}}, & \dot{\hat{\lambda}}_{p}^{i}=-\frac{\partial \hat{\mathbb{H}}^{O C}(\cdot)}{\partial \hat{p}_{i}} \\
0=g^{r}(x), & 0=\frac{\partial \hat{\mathbb{H}}^{O C}(\cdot)}{\partial y_{r}} \\
0 & =\frac{\partial \hat{\mathbb{H}}^{O C}(\cdot)}{\partial \hat{u}_{i}} &
\end{array}
$$

with the Hamiltonian being

$$
\hat{\mathbb{H}}^{O C}\left(x, \hat{p}, y, \hat{u}, \hat{\lambda}^{q}, \hat{\lambda}_{p}, \eta\right)=\hat{\lambda}_{i}^{q} \frac{\partial H^{M}}{\partial \hat{p}_{i}}+\hat{\lambda}_{p}^{i}\left(-\frac{\partial H^{M}}{\partial x^{i}}+\hat{u}_{i}\right)-\eta_{r} G^{r}(x, \hat{p}, y, \hat{u})-C(x, \hat{p}, y, \hat{u})
$$

whereat $G^{r}(x, \hat{p}, y, \hat{u})$ corresponds to the implicit definition of the dual normal quantity $y_{r}$. For index-3 DAEs one has

$$
G^{r}(x, \hat{p}, y, \hat{u})=\frac{\mathrm{d}^{2} g^{r}(x)}{\mathrm{d} t^{2}}
$$

\subsection{The optimal control problem in redundant coordinates}

Even though the proper necessary optimality conditions in redundant coordinates are given by [9] (see also [10]), the treatment of the boundary conditions for (OCBVPR) still demands further elaboration. Comparing (53) and (55), it can be seen that the natural boundary conditions nevertheless arise from integration by parts:

$$
\begin{aligned}
& \int_{t_{0}}^{t_{f}} \hat{\lambda}_{i}^{q} \delta \dot{x}^{i} \mathrm{~d} t=\left[\hat{\lambda}_{i}^{q} \delta x^{i}\right]_{t_{0}}^{t_{f}}-\int_{t_{0}}^{t_{f}} \dot{\hat{\lambda}}_{i}^{q} \delta x^{i} \mathrm{~d} t \\
& \int_{t_{0}}^{t_{f}} \hat{\lambda}_{p}^{i} \delta \dot{\hat{p}}_{i} \mathrm{~d} t=\left[\hat{\lambda}_{p}^{i} \delta p_{i}\right]_{t_{0}}^{t_{f}}-\int_{t_{0}}^{t_{f}} \dot{\hat{\lambda}}_{p}^{i} \delta \hat{p}_{i} \mathrm{~d} t
\end{aligned}
$$

and thus the proper optimal control BVP is initially given by the necessary optimalty conditions (54) along with the boundary conditions

$$
\left[\hat{\lambda}_{i}^{q} \delta x^{i}\right]_{t_{0}}^{t_{f}}=0 \quad\left[\hat{\lambda}_{p}^{i} \delta \hat{p}_{i}\right]_{t_{0}}^{t_{f}}=0
$$

However, since $p_{r}=0$ and $n^{r}(x)=$ const. has to hold everywhere and especially on the boundaries, the corresponding variations have to vanish. Making use of (19) and (20), the boundary conditions in redundant coordinates reduce to

$$
\begin{aligned}
& 0=\left[\hat{\lambda}_{i}^{q}\left(\frac{\partial x^{i}}{\partial n^{r}} \delta n^{r}+\frac{\partial x^{i}}{\partial q^{a}} \delta q^{a}\right)\right]_{t_{0}}^{t_{f}}=\left[\lambda_{r}^{q} \delta n^{r}+\lambda_{a}^{q} \delta q^{a}\right]_{t_{0}}^{t_{f}}=\left[\lambda_{a}^{q} \delta q^{a}\right]_{t_{0}}^{t_{f}} \\
& 0=\left[\hat{\lambda}_{p}^{i}\left(\frac{\partial n^{r}}{\partial x^{i}} \delta p_{r}+\frac{\partial q^{a}}{\partial x^{i}} \delta p_{a}\right)\right]_{t_{0}}^{t_{f}}=\left[\lambda_{p}^{r} \delta p_{r}+\lambda_{p}^{a} \delta p_{a}\right]_{t_{0}}^{t_{f}}=\left[\lambda_{p}^{a} \delta p_{a}\right]_{t_{0}}^{t_{f}}
\end{aligned}
$$

Accordingly, the proposed form of the optimal control BVP in redundant coordinates is given by the necessary optimality conditions (54) together with the boundary conditions

$$
0=\left[\hat{\lambda}_{i}^{q} \frac{\partial x^{i}}{\partial q^{a}} \delta q^{a}\right]_{t_{0}}^{t_{f}} \quad 0=\left[\lambda_{p}^{i} \frac{\partial q^{a}}{\partial x^{i}} \delta p_{a}\right]_{t_{0}}^{t_{f}}
$$




\subsection{Numerical example of the optimal control BVP}

Let the cost functionals be defined by

$$
C(x, \hat{p}, y, \hat{u})=\frac{1}{2} \hat{u}_{i} \hat{g}^{i j} \hat{u}_{j}, \quad C(q, p, u)=\frac{1}{2} u_{a} g^{i j} u_{a}
$$

with the metric tensors given by

$$
\hat{g}^{i j}=\hat{\mathbf{M}}^{-1}, \quad g^{a b}=\mathbf{M}^{-1}
$$

Using once more the midpoint rule for the discretization of the differential equations in the necessary optimality conditions in term of redundant coordinates yields

$$
\begin{aligned}
x_{n+1}^{i}-x_{n}^{i} & = & h \frac{\partial \hat{\mathbb{H}}^{O C}}{\partial \hat{\lambda}_{i}^{q,+\frac{1}{2}}} ; & n & =\{0,1, \ldots, N-1\} \\
p_{i}^{n+1}-p_{i}^{n} & = & h \frac{\partial \hat{H}^{O C}}{\partial \hat{\lambda}^{i}} ; & n & =\{0,1, \ldots, N-1\} \\
\hat{\lambda}_{i}^{q, n+1}-\hat{\lambda}_{i}^{q, n} & = & h\left(-\frac{\partial \hat{\mathbb{H}}^{O C}}{\partial x_{n+1}^{i}}\right) ; & n & =\{0,1, \ldots, N-1\} \\
\hat{\lambda}_{p, n+1}^{i}-\hat{\lambda}_{p, n}^{i} & = & h\left(-\frac{\partial \hat{\mathbb{H}}^{O C}}{\partial \hat{p}_{i}^{n+\frac{1}{2}}}\right) ; & n & =\{0,1, \ldots, N-1\} \\
0 & = & g^{r}\left(x_{n}^{i}\right) ; & n & =\{0,1, \ldots, N\} \\
0 & = & \frac{\partial \hat{\mathbb{H}}^{O C}(\cdot)}{\partial y_{r}^{n}} ; & n & =\{0,1, \ldots, N\} \\
0 & = & \frac{\partial \hat{H}^{O C}(\cdot)}{\partial \hat{u}_{i}^{n}} ; & n & =\{0,1, \ldots, N\}
\end{aligned}
$$

As already done in the context of the mechanical BVP, the boundary conditions may also be enforced by Lagranian multipliers. Consequently, we get

$$
\begin{array}{rlrl}
\frac{\partial q_{0}^{a}}{\partial x_{0}^{i}}\left(x_{0}^{i}-\bar{x}_{0}^{i}\right) \delta \mu_{a}^{0} & =0 ; & & \frac{\partial q_{N}^{a}}{\partial x_{N}^{N}}\left(x_{N}^{i}-\bar{x}_{N}^{i}\right) \delta \mu_{a}^{N}=0 \\
\frac{\partial x_{0}^{i}}{\partial q_{0}^{a}}\left(p_{i}^{0}-\bar{p}_{i}^{0}\right) \delta v_{0}^{a}=0 ; & & \frac{\partial x_{N}^{N}}{\partial q_{N}^{a}}\left(p_{i}^{N}-\bar{p}_{i}^{N}\right) \delta v_{N}^{a}=0 \\
\left(\frac{\partial x_{0}^{i}}{\partial q_{0}^{a}} \lambda_{i}^{q, 0}-\mu_{a}^{0}\right) \delta q_{0}^{a}=0 ; & & \left(\frac{\partial x_{N}^{i}}{\partial q_{N}^{a}} \lambda_{i}^{q, N}-\mu_{a}^{N}\right) \delta q_{N}^{a}=0 \\
\left(\frac{\partial q_{0}^{a}}{\partial x_{0}^{i}} \lambda_{p, 0}^{i}-v_{0}^{a}\right) \delta p_{a}^{0}=0 ; & & \left(\frac{\partial q_{N}^{a}}{\partial x_{N}^{i}} \lambda_{p, N}^{i}-v_{N}^{a}\right) \delta p_{a}^{N}=0
\end{array}
$$

together with the implicit definition of the dual quantities $y_{r}$ on the boundaries already known from the mechanical BVP given by (56).

In minimal coordinates, the discrete necessary optimality condition simplify to

$$
\begin{aligned}
& q_{n+1}^{a}-q_{n}^{a}=h \frac{\partial \mathbb{H}^{O C}}{\partial \lambda_{a}^{q, n+\frac{1}{2}}} ; \quad n=\{0,1, \ldots, N-1\} \\
& p_{a}^{n+1}-p_{a}^{n}=h \frac{\partial H^{O C}}{\partial \lambda_{p, n+\frac{1}{2}}^{a}} ; \quad n=\{0,1, \ldots, N-1\} \\
& \lambda_{a}^{q, n+1}-\lambda_{a}^{q, n}=h\left(-\frac{\partial \mathbb{H}^{o C^{2}}(\cdot)}{\partial q_{n+\frac{1}{2}}^{a}}\right) ; \quad n=\{0,1, \ldots, N-1\} \\
& \lambda_{p, n+1}^{i}-\lambda_{p, n}^{i}=h\left(-\frac{\partial \mathbb{H}^{O C}(\cdot)}{\partial p_{i}^{n+\frac{1}{2}}}\right) ; \quad n=\{0,1, \ldots, N-1\} \\
& 0 \quad=\quad \frac{\partial \mathbb{H}^{O C}(\cdot)}{\partial u_{a}^{n}} ; \quad n=\{0,1, \ldots, N\}
\end{aligned}
$$

along with the boundary conditions

$$
\begin{aligned}
\left(q_{0}^{a}-\bar{q}_{0}^{a}\right) \delta \mu_{a}^{0} & =0 ; & & \left(q_{N}^{a}-\bar{q}_{N}^{a}\right) \delta \mu_{a}^{N}=0 ; \\
\left(p_{a}^{0}-\bar{p}_{a}^{0}\right) \delta v_{0}^{a} & =0 ; & & \left(p_{a}^{N}-\bar{p}_{a}^{N}\right) \delta v_{N}^{a}=0 ; \\
\left(\lambda_{a}^{q, 0}-\mu_{a}^{0}\right) \delta q_{0}^{a} & =0 ; & \left(\lambda_{a}^{q, N}-\mu_{a}^{N}\right) \delta q_{N}^{a} & =0 ; \\
\left(\lambda_{p, 0}^{a}-v_{0}^{a}\right) \delta p_{a}^{0} & =0 ; & \left(\lambda_{p, N}^{a}-v_{N}^{a}\right) \delta p_{a}^{N} & =0 ;
\end{aligned}
$$




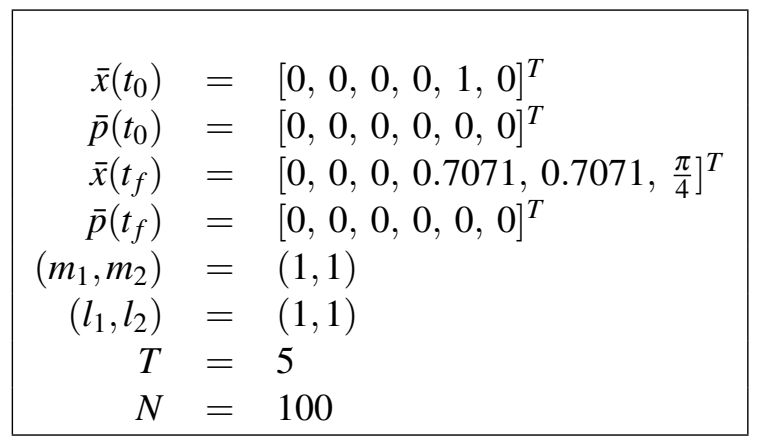

Table 2. Specified boundary conditions as well as physical and geometric parameters of the mechanical system in the optimal control problem
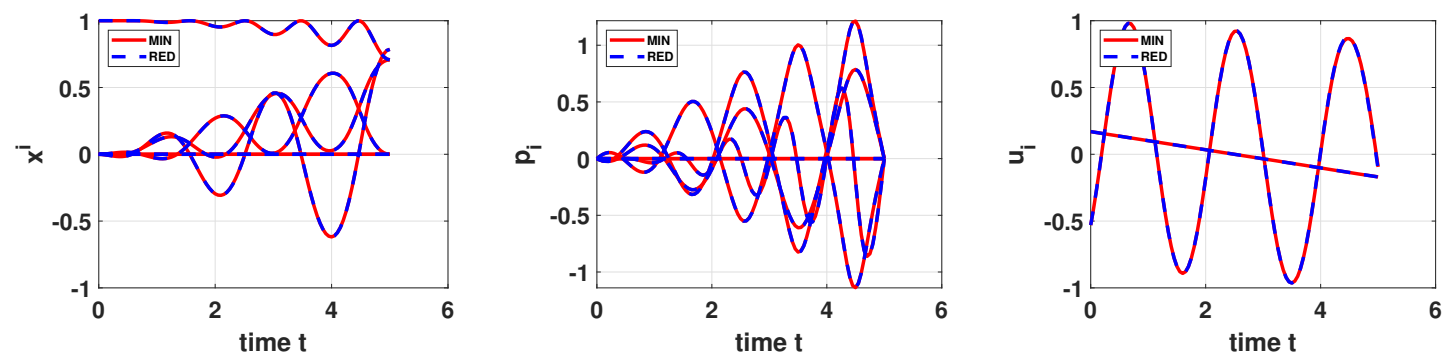

Figure 4. Solutions of the optimal control problem boundary value problem defined by Table 2. Here the positions (left) and momenta (center) are shown in redundant coordinates while the redundant controls (right) are shown in minimal coordinates.

The boundary conditions chosen for the optimal control example, the length and mass of the slide and the pendulum as well as the length of the time interval $I \in[0, T]$ and the number of discrete time intervals, $N$, is shown in Table 2.

Comparing the solutions depicted in Fig. 4, it can be seen that the two BVP under consideration are indeed equivalent.

Fig. 5 also shows a sequence of the solution of the optimal control boundary value problem.

\section{CONCLUSION}

In this paper, the boundary value problem of the optimal control problem in redundant coordinates was considered in more detail. Due to the mathematical similarity of the Lagrange formalism of classical mechanics and the optimal control problem, the first step was to consider the boundary
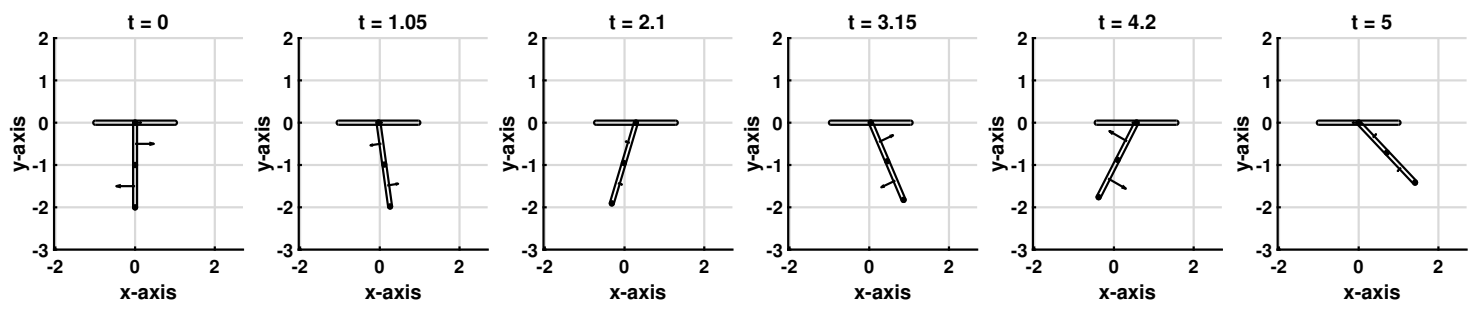

Figure 5. Sequence of the motion of the controlled mechanical system defined by the optimal control BVP with the data given in Table 2. 
value problem in redundant coordinates in the simplified framework of classical mechanics. After the requirements on the variations on the boundary, which occur due to the algebraic constraints imposed on the redundant coordinates, were discussed on the mechanical level, the knowledge gained from this was transferred to the optimal control problem. Here, the challenging optimal control problem in redundant coordinates was first discussed and the correct necessary optimality conditions were referred to. Subsequently, with the help of the clear separation of the coordinates into normal and tangential parts, which was already known from the mechanical boundary value problem, the optimal control problem was formulated in redundant coordinates. Finally, it was verified by means of a numerical example that the optimal control problem formulated in both redundant and minimal coordinates leads to equivalent numerical results.

\section{ACKNOWLEDGEMENTS}

This work was funded by the Deutsche Forschungsgemeinschaft (DFG, German Research Foundation) - project number 442997215. This support is gratefully acknowledged.

\section{REFERENCES}

[1] Blajer, W.: An orthonormal tangent space method for constrained multibody systems. Comput. Methods Appl. Mech. Engrg. 121 (1995) 45-57

[2] Arnol'd, V.: Mathematical Methods of Classical Mechanics. Springer (1989) doi: 10.1007/978-1-4757-2063-1.

[3] Livens, G.: On Hamilton's principle and the modified function in analytical dynamics. Proceedings of the Royal Society Edinburgh 39(IX) (1919) 113-119 doi: $10.1017 /$ S0370164600018617.

[4] Bou-Rabee, N.: Hamilton-Pontryagin integrators on Lie groups. PhD thesis, California Insitute of Technology (2007)

[5] Gear, C.W. Leimkuhler, B., Gupta, G.: Automatic integration of Euler-Lagrange equations with constraints. Journal of Computational and Applied Mathematics 12-13 (1985) 77-90

[6] Betsch, P.: The discrete null space method for the energy consistent integration of constrained mechanical systems part I: Holonomic constraints. Comput. Methods Appl. Mech. Engrg. 194 (2005) 5159-5190

[7] Betsch, P., Leyendecker, S.: The discrete null space method for the energy consistent integration of constrained mechanical systems. part II: Multibody dynamics. Int. J. Numer. Meth. Engng 67 (2006) 499-552 doi: 10.1002/nme.1639.

[8] Ober-Blöbaum, S.: Discrete Mechanics and Optimal Control. PhD thesis, Paderborn (2008)

[9] Roubíček, T., Valášek, M.: Optimal control of causal differential-algebraic systems. J. Math. Anal. Appl. 269 (2002) 616-641

[10] Gerdts, M.: Optimal Control of ODEs and DAEs. De Gruyter, Berlin, Boston (2011) ISBN 978-3-11-024995-8.

[11] Betsch, P., Becker, C.: Conservation of generalized momentum maps in mechanical optimal control problems with symmetry. Int. J. Numer. Meth. Engng 111(2) (2017) 144-175 doi: $10.1002 / \mathrm{nme} .5459$. 